\title{
Erratum: Quantum model for mode locking in pulsed semiconductor quantum dots [Phys. Rev. B 94, 245308 (2016)]
}

\author{
W. Beugeling, Götz S. Uhrig, and Frithjof B. Anders \\ (Received 6 November 2017; published 21 November 2017)
}

\section{DOI: 10.1103/PhysRevB.96.199904}

The values of the transverse components of the Overhauser field in Figs. 2(e) and 2(f) have been plotted with an incorrect scaling factor on the vertical axis. The correct multiplier on the vertical axis should be $10^{-6}$ instead of $10^{-3}$.

The numbers on the vertical axis in Fig. 3(a), representing the peak growth rate $\eta_{t}$, do not align properly with the markers. The correct values should run from -40 (bottom) to 20 (top).

Below, we provide the corrected figures for reference. Since we have not made quantitative statements based directly on the values represented by the affected figures, the other results and conclusions of the article remain valid without change.
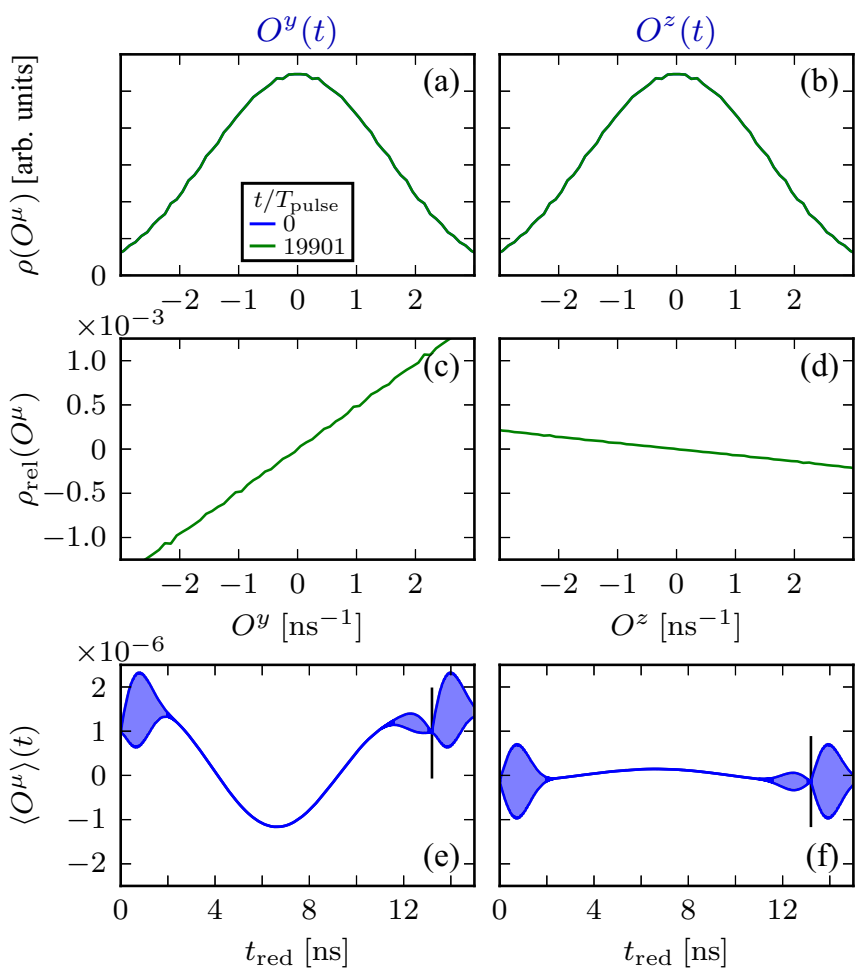

FIG. 2. Behavior of the transverse components $O^{\mu}(\mu=y, z)$ after many pulses $\left(t=19901 T_{\text {pulse }}\right)$. (a), (b) Probability distributions of the observables in their respective eigenbases. (c), (d) Relative differences $\rho_{\text {rel }}$ of the distribution at $t=19901 T_{\text {pulse }}$ with the initial one. (e), (f) Time evolution $O^{\mu}(t)$ after $19901 T_{\text {pulse }}$ with $t_{\text {red }} \equiv$ $t-19901 T_{\text {pulse }}$. The shaded regions indicate fast oscillations with frequency close to the Larmor frequency $\lambda$. The nuclear couplings have been chosen such that $T^{*}=1 \mathrm{~ns}$.

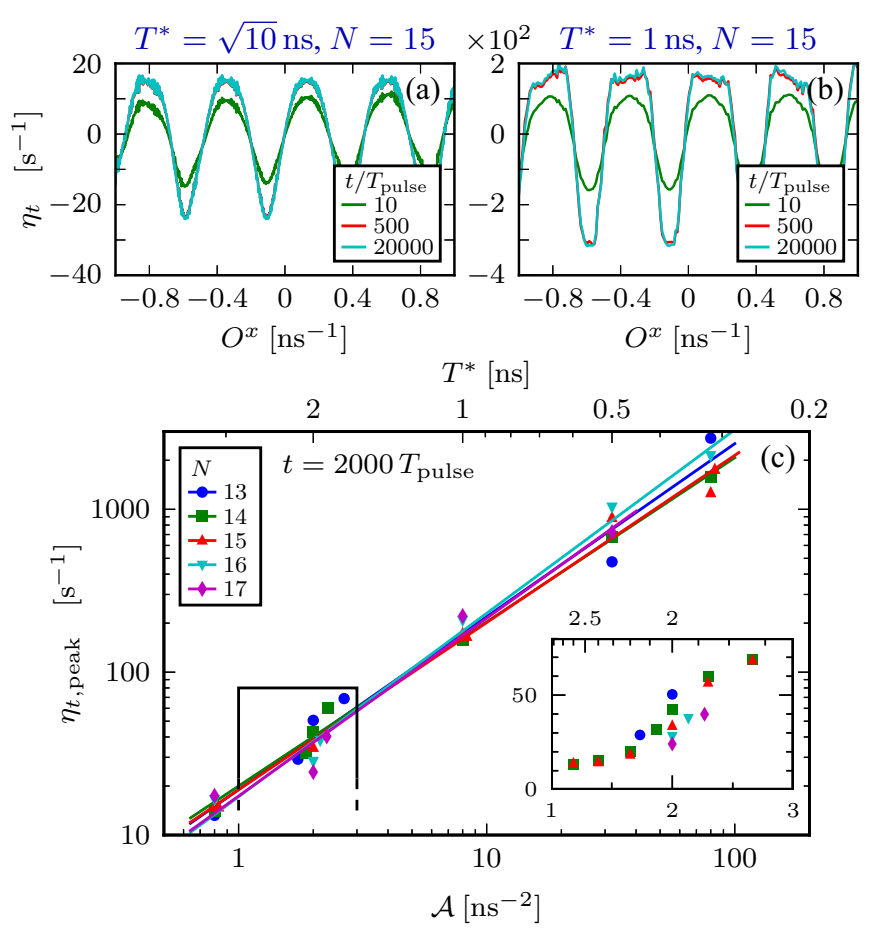

FIG. 3. (a) Growth rate $\eta_{t}=\rho_{\text {rel }} / t$ for couplings scaled such that $T^{*}=3.16 \mathrm{~ns}$ (a) or $T^{*}=1 \mathrm{~ns}$ (b). (c) Dependence of the peak heights of $\rho_{\text {rel }}$ on $\mathcal{A}$ for different $N$ 's. The lower horizontal axis shows $\mathcal{A}$, and the upper horizontal axis the equivalent dephasing times $T^{*}$. For each $N$, we fit power laws $\propto \mathcal{A}^{\alpha}$ with $\alpha \approx 1$, depicted as straight lines. In the inset, we zoom in on the regime around $T^{*}=2 \mathrm{~ns}$. The axes of the inset are linear. 